\title{
Current research status on the psychological situation of parents of children with congenital heart disease
}

\author{
Sabina Biber ${ }^{1}$, Caroline Andonian ${ }^{1,2}$, Jürgen Beckmann ${ }^{2,3}$, Peter Ewert ${ }^{2}$, Sebastian Freilinger ${ }^{2}$, \\ Nicole Nagdyman ${ }^{2}$, Harald Kaemmerer ${ }^{2}$, Renate Oberhoffer ${ }^{1,2}$, Lars Pieper ${ }^{4}$, Rhoia Clara Neidenbach ${ }^{2}$ \\ ${ }^{1}$ Department of Sport and Health Sciences, Technical University of Munich, Munich, Germany; ${ }^{2}$ Department of Paediatric Cardiology and Congenital \\ Heart Defects, German Heart Centre Munich, Munich, Germany; ${ }^{3}$ School of Human Movement and Nutrition Sciences, University of Queensland, \\ Brisbane, Australia; ${ }^{4}$ Faculty of Psychology, Institute of Clinical Psychology and Psychotherapy, Technical University Dresden, Germany \\ Contributions: (I) Conception and design: All authors; (II) Administrative support: All authors; (III) Provision of study materials or patients: All \\ authors; (IV) Collection and assembly of data: All authors; (V) Data analysis and interpretation: All authors; (VI) Manuscript writing: All Authors; \\ (VII) Final approval of manuscript: All Authors. \\ Correspondence to: Sabina Biber, B.Ed., Chair of Sport Psychology, Department of Sport and Health Sciences, Technical University of Munich, \\ Munich, Germany. Email: sabina.biber@tum.de.
}

\begin{abstract}
Congenital heart diseases (CHD) are a leading cause of morbidity in children with a high impact on the psychological health of parents. Possible short-term and long-term psychological problems among parents are addressed in the current paper. The diagnosis of CHD paired with subsequent surgical and interventional treatment and prolonged hospital stays cause acute psychological distress and can lead to posttraumatic stress disorders (PTSD). As the disease course progresses, the impact on parents' health tends to decrease, but the risk of developing long-term psychological issues remains high. Studies have focused mainly on stress and other distressing symptoms without explicitly addressing the effects of a CHD diagnosis on the family system. Since the social environment may play an important role in parent's life, it may be useful to conduct studies to address these issues. In particular, the psychological situation of the father and the impact of the child's disease on the different dimensions of the father's life, such as parenting skills and influences on the parental relationship, have been largely neglected. Recent research has also disregarded the impact of CHD on siblings of the affected child. Research on chronic diseases in general has shown that the children's age and severity of the disease are related to an increased level of stress. Given the severity of CHD, anxiety and depression were higher in parents with children with more severe conditions. In addition, the results suggest that a positive construction of the parent-child relationship (attachment and bonding) is impaired, especially in mothers. Mothers reported worries and concerns about the challenging tasks they would face after learning about their child's CHD and how they can deal with their child's needs. It has also been shown that the child's illness has a negative impact on the whole family system, including the parent's relationship. Impairments on the parental relationship were perceived differently among mothers and fathers. Thus, there is high need for major changes to be identified, developed and implemented in the psychological care of parents with chronically ill children. So far, research has focused more on the psychological status of parents with chronically ill children, but less research has closely examined the effects of a child's CHD on its parent's mental health even though there is a high demand in additional support. A holistic treatment approach should include professional parental support, especially during children's hospitalization, information on the home care resources and services (especially respite services) and psychological support for parents.
\end{abstract}

Keywords: Congenital heart defects (CHD); impact parents; parents with children with CHD; psychological situation; mental health; chronic disease

Submitted Apr 05, 2019. Accepted for publication Jul 09, 2019.

doi: $10.21037 / \mathrm{cdt} .2019 .07 .07$

View this article at: http://dx.doi.org/10.21037/cdt.2019.07.07 


\section{Introduction}

Congenital heart disease (CHD) is categorized as the most common type of birth defects (1). Worldwide, the prevalence of CHD is estimated at about 8 per 1,000 live births $(2,3)$. According to the PAN study (4), the prevalence of children born with CHD in Germany in 2007 was $1.08 \%$. Soon after birth, babies with a critical state of health due to CHD must undergo surgery or interventional treatment-in many cases as early as the first year of life (5). Today, however, $95 \%$ of these children reach adulthood as a result of the success of modern treatment options. Although there has been remarkable progress in disease management, the performance and physical abilities vary enormously due to a variety of CHD types. These CHD types include simple, moderate and complex heart defects. Evidence suggests that the amount of complex CHD, requiring hospital treatment, increased significantly within 1995 and 2015, as shown in Figure 1. This increase could have an impact on disease management due to the surge of complications and other related difficulties (6). In addition, managing this complex, chronic disease is a major stress factor in parent's lives. The management of various interventions, hospital stays and the constant need to deal with uncertain prognostics absorbs a substantial part of parents' lives and induces high levels of psychological stress. The aim of this review is to summarize current findings on the psychological status of parents of children with CHD, point out the necessity of further research and discuss relevant clinical measures.

\section{Prevalence of psychological issues among parents of children with CHD}

Short- and long-term psychological issues among parents of children with CHD have been discussed (7-11). Critical life events for the parents include the CHD diagnosis and subsequent interventions leading to acute psychological problems (7). A longitudinal study by Lawoko and Soares (11) showed persistent psychological problems in $7-22 \%$ of the participating parents. Among the specific mental health problems assessed during the study, prevalence rates above those of the general population were found for depression (18\%), anxiety (16-18\%), somatization (31-38\%), and hopelessness (16\%). Also, Woolf-King et $a l$. showed in their systematic review of 30 studies, that $25 \%$ to $50 \%$ of the parents of children with congenital heart defect indicated elevated symptoms of depression and/or anxiety. About $30 \%$ to $80 \%$ of the CHD parents experienced severe psychological distress (12). Figure 2 shows prevalence rates of depression in mothers extracted from Woolf-King et al.'s review (8,13-16). The comparability of the prevalences is limited as measurement points of depression slightly differ among the studies.

Rao et al. (17) conducted an evaluation on psychopathology and coping mechanisms in 30 parents of children with a chronic hereditary disease using the SCL-90-R and Mechanisms of Coping scales. General distress among parents was found to be very high $(86.7 \%)$.

Hearps et al. (8) described that the majority of parents can adapt to acute stress following children's surgery. However, $38.5 \%$ of the parents were identified with a persisting high psychological risk which is associated with substantial emotional distress (8). Acute posttraumatic stress disorder (PTSD) was found among $16.4 \%$ of mothers and $13.3 \%$ of fathers after discharge of their CHD children (9). Another study assessed PTSD in $19 \%$ of parents of children who underwent heart transplantation (10). Several prevalences of acute PTSD are shown in Figure 3 (insert sources).

\section{Psychological distress in parents of children with CHD}

While research has frequently addressed the psychological burden on parents of children with severe diseases such as cancer, fewer studies have been conducted on the impact of paediatric CHD on the mental health and well-being of CHD parents.

Most research on CHD parents focuses on quality of life (QoL) in general (18). Sileshi et al. (19) found a significantly lower QoL in CHD mothers compared to the control group using the Short Form Health (SF36) questionnaire in a study with 135 CHD mothers in Ethiopia. Soulvie et al. (20) reviewed 25 studies on psychological distress experienced by parents of young CHD children. The authors focused on the following dimensions of psychological stress: bonding difficulties between child and parents, concern, anxiety and depression and other manifestations of stress. Based on the categorization in this report, additional literature on various aspects of health and well-being in CHD parents is presented in the following section.

\section{Stress}

According to studies the children's age has an effect on the parent's level of stress (20). Mothers have reported 
Overall changing prevalence in $\%$ of $\mathrm{CHD}$ complexity

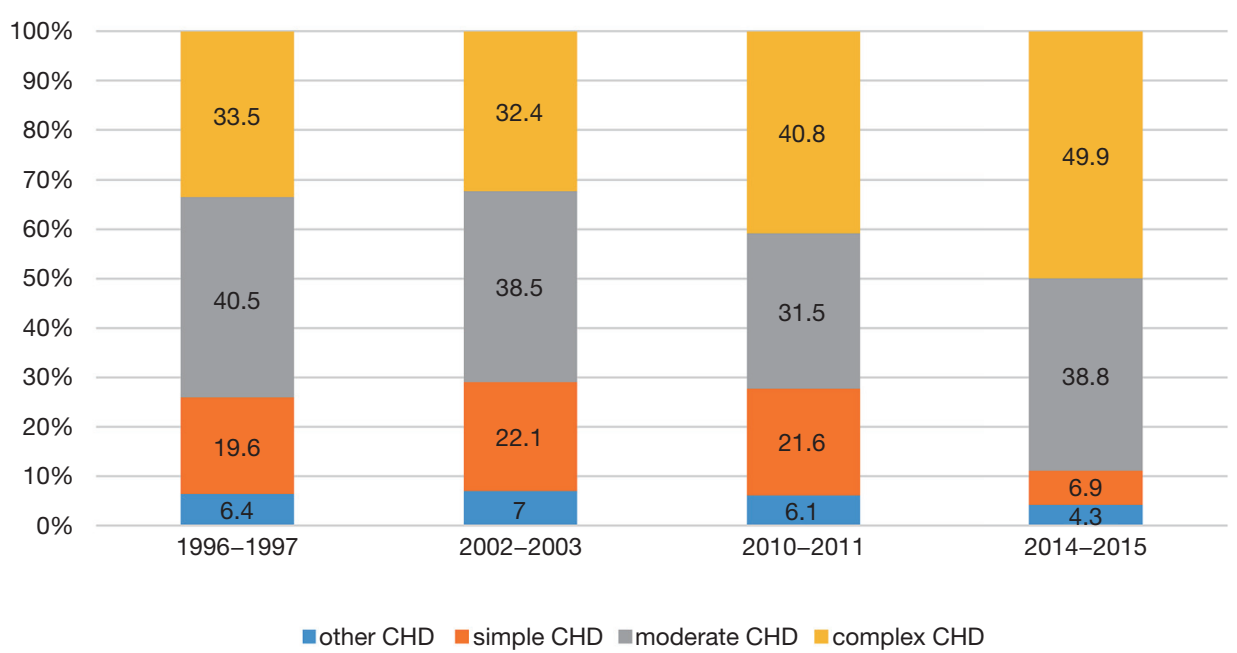

Figure 1 Overall changing prevalence of CHD complexity in \% of children with CHD who required hospital treatment from 1996-2015 (6). $\mathrm{CHD}$, congenital heart diseases.

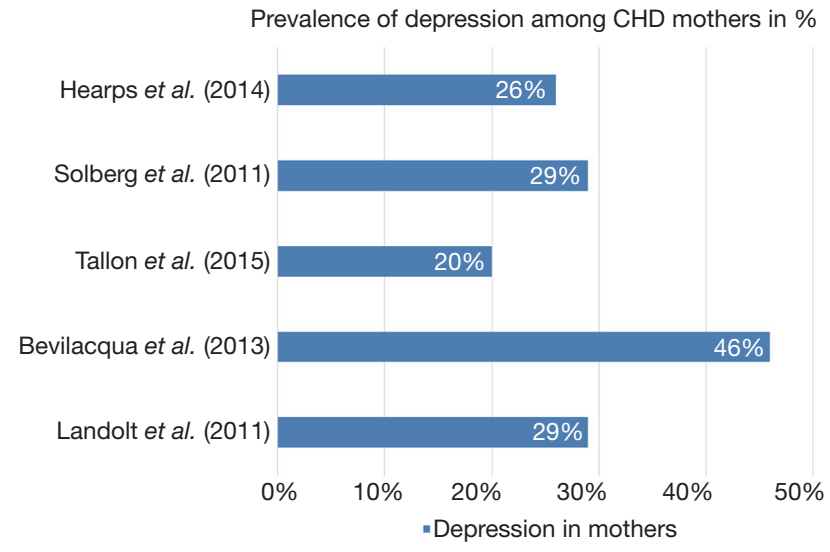

Figure 2 Prevalence of depression among CHD mothers in \% (8, 13-16). CHD, congenital heart diseases.

difficulties in establishing mutual attachment to their infants, particularly in relation to the feeding process (21). All mothers in this study reported, that the feeding process was a source of distress for them. Also, ambiguous infant responses to maternal cues led to a feeling of frustration in the examined mothers (21). Consequently, mothers developed coping strategies such as denying infant difficulties like poor feeding or delayed growth to protect self-esteem (21).

Stress experience of parents of CHD children remains continuously increased even at preschool age (20). According to the findings of Majnemer et al. (22), parents

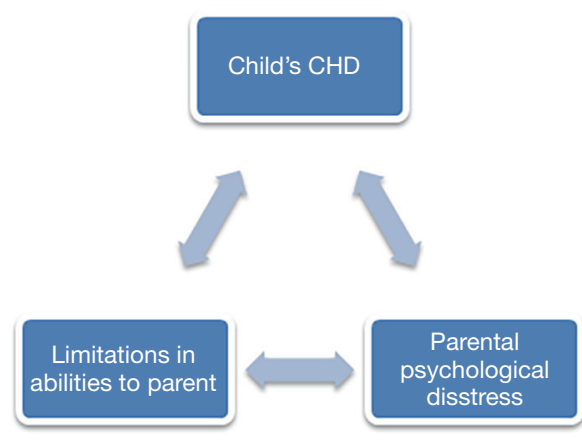

Figure 3 Bi-directional effects of a child's CHD. CHD, congenital heart diseases.

of five-year-old CHD children reported stress or feelings of defensiveness towards their child, especially if the child showed challenging behavior.

Lee et al. (23) found parenting stress in mothers of children with CHD to be significantly related to the uncertainty of perceived social support, ambiguity, lack of clarity and lack of information (23). Ambiguity refers to the uncertainties regarding the care of the child. Lack of clarity is related to insufficient information about the child's treatment and lack of information being defined as missing information about the diagnosis and sincerity of the disease. Accordingly, mothers with higher social support and less 
insecurity stated lower parenting stress (23).

Typically, there are bi-directional effects which are depicted in Figure 3: parental stress is very likely to have negative effects on the children themselves (20). Simultaneously, the child's disease negatively impacts the parent's mental health and can potentially compromise their parenting abilities which may in turn have negative effects on the child's defect.

Due to the heterogeneity of CHD, the presence and levels of stress may vary according to the severity of the disease. A study conducted by the Medical College of Wisconsin from Brosig et al. on psychosocial outcomes for preschoolers and families after surgery for complex CHD detected significant differences between two samples with CHD: parents of children with hypoplastic left heart syndrome (HLHS) described higher overall stress levels compared to parents of children with transposition of the great arteries (TGA). In addition, a higher burden was observed in terms of parental competence. Furthermore, positive correlations were obtained for parental stress and perception of the overall impact of the disease on the family ( $\mathrm{r}=0.44, \mathrm{P}=0.037)$ as well as personal strain $(\mathrm{r}=0.49$, $\mathrm{P}=0.017$ ). Compared to test norms, the HLHS group showed higher scores on child demandingness, which made parenting more difficult and lower values on parental attachment, but also lower values on depression were found in this study (24).

In sum, the prevalence of stress among CHD parents is related to the management of the disease and several factors determine the level of stress to which parents are exposed. It is therefore necessary to examine potential stressors such as the severity of the defect, social support, stability of the parent's relationship and resources and coping strategies to provide the best possible support.

\section{Parent-child interactions and relationship issues}

Relationship difficulties are already recognized in the literature in the infant phase, resulting from the effects of the child's CHD on the parent-child bonding process and from disruptive factors in the caregiving process (20). A study by Gardner et al. (25) revealed that the influence and involvement of mothers in children with CHD is more diverse than in a control group. Ambiguous child responses to maternal engagement hinder the establishment of a stable mother-infant relationship.

Darke and Goldberg conducted one of the very few studies to investigate the caregiving experiences of CHD fathers. Less positive behavior towards the child was observed in fathers who reported high levels of stress in relation to handling the child's difficulties, parental competence and connections to the child (26).

Goldberg et al. (27) examined the infant-mother relationship in 42 infants with $\mathrm{CHD}$. It was found that compared to healthy controls, significantly fewer children with CHD had a secure relationship with their mothers. However, the quality of the relationship did not depend on the mother's personal stress or psychological well-being. Interestingly, the severity of the defect did not directly affect the quality of the infant-mother relationship, but the security of a quality infant-mother relationship had a positive impact on the improvement of children's health compared to that observed in insecure relationships.

In summary, only a few studies have focused on interactions and relationships between parents and CHD children. In general, results stress the importance of a stable relationship as it may have reciprocal impact on child and parents. An unstable relationship might interfere with the child-parenting bonding and may have a negative effect on the future relationship. In general, evidence suggests difficulties in the parent-child connection as a result of CHD. It is essential to further examine factors that may influence these changes and consider the underlying factors of the parent-child bonding such as insecurities in infants' upbringing and acceptance of the extraordinary circumstances.

\section{Worry and concern}

Worry and concern among CHD parents have been studied less often than stress. However, Soulvie et al. described the prevalence of worry and concern in CHD parents as fairly high (24,28-31). In Maxwell et al.'s research (29), 90\% of the 150 parents examined showed a high level of worry at the time of diagnosis, $38 \%$ also expressed worry about the child's siblings. Additional findings by Brosig et al. support that $71 \%$ of parents of children with HLHS and TGA $(n=26)$ reported concerns about their child's future (24).

Docherty et al. (31) investigated different aspects of worry in relation to mothers with children with different disorders such as: chronic lung diseases, CHD and other chronic diseases. Eighty-three mothers of medically fragile infants participated in the study. Using the Child Health Worry Scale (CHWS), their results suggest that these mothers have moderately high level of worry about their child's disease. More worry was observed about the present 
and future health of the child, the care process at home, compared to concerns about the possible death of the child.

In her research Pinelli addressed mother's concerns after bringing their child home from hospital. The results of interviews showed that the majority of the concerns are related to the tasks of learning anticipation and recognizing the baby's needs (30). These results indicate that more information on how to deal with the child and its disease should be provided before the child is brought home from hospital.

\section{Anxiety and depression}

Anxiety and depression have been in the focus of various, recent studies, in order to investigate changes in the psychological state of parents with children with chronic diseases $(11,24,32)$.

Studies using the Parenting Stress Index (PSI) showed that parents of sick infants reported higher rates of depression and a reduced sense of competence compared to a group of parents with healthy children. The PSI is divided into three major domains of stress: child characteristics, parent characteristics and situational/demographic life stress. The parent domain focuses on aspects such as isolation, health and spouse/parent relationship, while the child domain covers topics such as demandingness, child mood, child adaptability to environmental changes and other criteria (33).

In the child domain of the PSI (distractibility/ hyperactivity and reinforcement of parent) fathers stated more stress than mothers. In contrast, in the parent domain, mothers claimed more stress than fathers on six of the seven scales (all excluding social isolation) (27).

As a noticeable limitation Goldberg et al. (34) did not critically question the circumstances of parenthood-it is, therefore, unclear which one of the parents was mainly involved in the childcare process, which could be a factor influencing the perception of parental mental health.

Brosig et al. (24) used the Brief Symptom Inventory (BSI) to assess differences in parents' General Severity Index (GSI) scores depending on more and less severe forms of CHD.

The BSI serves as a tool to get an overview of patient's symptoms and intensity at a given time. To quantify the patient's severity-of-illness, which is a part of the BSI, the GSI served as a measurement instrument. The results show that parents with children with more severe lesions had higher GSI scores, which is an indicator for an increased level of psychopathology (32).

\section{Other distressing emotions}

In addition to higher levels of anxiety and depression in CHD parents, they reported lower confidence in their abilities to parent $(34,35)$.

In interviews conducted by Brosig et al. (32) parents reported feelings of anger, helplessness, disbelief and doubt. These interviews were performed prenatally and six months after the birth of the baby.

Even though the emotional state of the parents improved six months after birth, a large number of parents reported difficulties in the experience of parenting. Particularly parents with HLHS expressed such problems. For example, one father stated: "There was no way to prepare for how hard this would be. It's been a constant adjustment.".

As described above parents' emotions are very likely to differ. Addressing these problems is a particular challenge for health professionals due to the fact that as an individual approach for each parent is essential to prevent psychological burdens. Furthermore, healthcare professionals are not sufficiently trained to identify parent's needs for psychological support.

In addition, a lack of staff capacity impairs adequate parental support, which would be necessary to prepare parents for the special needs of their child at home.

\section{Social impact}

Azhar et al. (36) investigated the impact of CHD on the social environment and QoL of patients and their families in Saudi Arabia. In their research 58.3\% of the participants reported to have received psychological support from their child's physician. Regarding explanations concerning the child's disease, $53.3 \%$ stated that they needed further information and instruction (36). As this research was conducted in Saudi Arabia, differences regarding social conditions in comparison to western countries should be taken into account.

Therefore, additional information and education from healthcare professionals is needed. In Sweden, Lawoko and Soares (37) investigated the social support of parents of children with CHD, other diseases and parents of healthy children. Comparing these three groups, the main results indicate that all participating mothers had a lower availability of social support compared to fathers.

The lowest availability of social support was observed within the group of mothers of children with CHD. Further analysis showed that factors such as care giving, 
financial stability, psychological distress and hopelessness could explain the variation in accessibility of social support rather than gender. These results indicate that parents of children with CHD are particularly at risk of developing psychological disorders due to a lack of social support and should, therefore, receive additional support.

Research findings from Hoekstra-Weebers et al. (38) should be taken into account when planning support of a family with a CHD child. In a study with parents of children with diagnosed cancer, these authors found a decline of social support over time. One hundred and twenty-eight parents reported their levels of psychological stress, quantity of support and dissatisfaction with support at three time-points: at diagnosis, at 6 and 12 months (38).

When coping with the more chronic stress at a later stage of the diagnosis, parents received less social support during that period. Consequently, the levels of distress on parents varies over time as does the social support, that should be taken into consideration when planning support for parents (38).

According to the heterogeneity of CHD, differences between the different forms and their impact on families were examined. In another study conducted in Saudi Arabia, a sample of 41 children diagnosed with CHD was split in two groups: group one with simple CHD and group two with complex CHD. Significantly higher impact on family scale (IFS), which includes the dimensions of financial burden, familial/social impact, personal strain and mastery was observed in families with complex CHD in comparison to families with children with minor heart disease (39).

\section{Parental relationship}

The diagnosis of a severe illness such as cancer or CHD must be considered as a critical life event for the child and the parents. Parents are faced with life-changing decisions, a shifted focus on the child and its disease and many other stress factors. No research has yet been conducted on the consequences of a CHD diagnosis for affected parents. Only few studies of parents with children with other chronic diseases investigated the impact on the parents' relationship.

In one study, Wiener et al. (40) examined the perceived influence of childhood cancer on parental relationships. Ninety-two parents participated, of whom $40 \%$ claimed they felt their relationship was moving in a negative direction. They identified the diagnosis and a relapse of the disease as the most stressful events with severe consequences for their relationship. Whereas parents felt most emotionally connected when they first learned of the diagnosis, they felt least connected to the child at the start and end of the treatment. The majority of the participants showed interest in counselling in order to address ways to foster their relationship (40).

Burns et al. (41) identified 47 couples with children with acute lymphoblastic leukemia (ALL) in a Canadian research project. Parental well-being and adjustment to the diagnosis of ALL were assessed at two time points: at diagnosis and two years after. Twenty-three percent of parents claimed crucial marital stress at the time of the diagnosis $(25.2 \%$ of the mothers and $21.3 \%$ of the fathers). The perceived marital stress ratings increased over time $(36.2 \%$ of mothers and $42.6 \%$ of fathers) (41). This demonstrates that the perception of the relationship may differ between the partners, which should be considered when advising parents with diseased children.

\section{Conclusions}

This article reviews the literature on the effects of diagnosis and treatment of a severe disease on the parents' health and well-being with a special focus on CHD.

To date, research findings regarding psychosocial effects of CHD are scarce. Various variables such as distress, depression, anxiety, relationship, worry and concern and others with an impact on CHD parents' mental health have been investigated but the evidence is still limited.

In general, the prevalence of psychological issues among affected parents is high. The majority of the studies examined the impact of the children's disease only on the health condition of the mother. Consequently, the father's perspectives were often not considered, as well as the influence on the parent's relationship. Social factors, like isolation from the communities or other activities were mostly not taken into account.

Consequently, further systematic research, in particular longitudinal and intercultural studies, is needed to develop interventions for parents. Teaching programs, extended psychological support and information material are urgently needed. In particular, support in terms of the parental relationship is demanded (40).

\section{Acknowledgments}

Funding: The authors would like to thank the "Deutsche Herzstiftung" as well as the patient organization "Herzkind e.V." for research funding (No. F-30-15) in the field 
of ACHD.

\section{Footnote}

Conflicts of Interest: The authors have no conflicts of interest to declare.

Ethical Statement: The authors are accountable for all aspects of the work in ensuring that questions related to the accuracy or integrity of any part of the work are appropriately investigated and resolved.

\section{References}

1. Centers for Disease Control and Prevention. Congenital Heart Defects. What are Congenital Heart Defects? Accessed 10-27 2018. Available online: https://www.cdc. gov/ncbddd/heartdefects/facts.html

2. van der Linde D, Konings EE, Slager MA, et al. Birth prevalence of congenital heart disease worldwide: a systematic review and meta-analysis. J Am Coll Cardiol 2011;58:2241-7.

3. Mason CA, Kirby RS, Sever LE, et al. Prevalence is the preferred measure of frequency of birth defects. Birth Defects Res A Clin Mol Teratol 2005;73:690-2.

4. Lindinger A, Schwedler G, Hense HW. Prevalence of congenital heart defects in newborns in Germany: Results of the first registration year of the PAN Study (July 2006 to June 2007). Klin Padiatr 2010;222:321-6.

5. National Heart Lung, and Blood Institute; National Institutes of Health; U.S. Department of Health and Human Services. 2018. Accessed 10-25 2018. Available online: https://www.nhlbi.nih.gov/health-topics/ congenital-heart-defects

6. Pfitzer C, Helm PC, Ferentzi H, et al. Changing prevalence of severe congenital heart disease: Results from the National Register for Congenital Heart Defects in Germany. Congenit Heart Dis 2017;12:787-93.

7. Kolaitis GA, Meentken MG, Utens EMWJ. Mental Health Problems in Parents of Children with Congenital Heart Disease. Front Pediatr 2017;5:102.

8. Hearps SJ, McCarthy MC, Muscara F, et al. Psychosocial risk in families of infants undergoing surgery for a serious congenital heart disease. Cardiol Young 2014;24:632-9.

9. Helfricht S, Latal B, Fischer JE, et al. Surgery-related posttraumatic stress disorder in parents of children undergoing cardiopulmonary bypass surgery: a prospective cohort study. Pediatr Crit Care Med 2008;9:217-23.
10. Farley LM, DeMaso DR, D'Angelo E, et al. Parenting stress and parental post-traumatic stress disorder in families after pediatric heart transplantation. J Heart Lung Transplant 2007;26:120-6.

11. Lawoko S, Soares JJ. Distress and hopelessness among parents of children with congenital heart disease, parents of children with other diseases, and parents of healthy children. J Psychosom Res 2002;52:193-208.

12. Woolf-King SE, Anger A, Arnold EA, et al. Mental health among parents of children with critical congenital heart defects: a systematic review. J Am Heart Assoc 2017;6:e004862.

13. 13 Solberg Ø, Dale MT, Holmstrøm H, et al. Emotional reactivity in infants with congenital heart defects and maternal symptoms of postnatal depression. Arch Womens Ment Health 2011;14:487-92.

14. Tallon MM, Kendall GE, Snider PD. Development of a measure for maternal confidence in knowledge and understanding and examination of psychosocial influences at the time of a child's heart surgery. J Spec Pediatr Nurs 2015;20:36-48.

15. Bevilacqua F, Palatta S, Mirante N, et al. Birth of a child with congenital heart disease: emotional reactions of mothers and fathers according to time of diagnosis. $\mathrm{J}$ Matern Fetal Neonatal Med 2013;26:1249-53.

16. Landolt MA, Buechel EV, Latal B. Predictors of parental quality of life after child open heart surgery: a 6-month prospective study. J Pediatr 2011;158:37-43.

17. Rao P, Pradhan PV, Shah H. Psychopathology and coping in parents of chronically ill children.Indian J Pediatr 2004;71:695-9.

18. Gregory MRB, Prouhet PM, Russell CL, et al. Quality of Life for Parents of Children With Congenital Heart Defect: J Cardiovasc Nurs 2018;33:363-71.

19. Sileshi L, Tefera E. Health-related quality of life of mothers of children with congenital heart disease in a subSaharan setting: cross-sectional comparative study. BMC Res Notes 2017;10:513.

20. Soulvie MA, Desai PP, White CP, et al. Psychological distress experienced by parents of young children with congenital heart defects: A comprehensive review of literature. J Soc Serv Res 2012;38:484-502.

21. Gudermuth S. Mothers' reports of early experiences of infants with congenital heart disease. Matern Child Nurs J 1975;4:155-64.

22. Majnemer A, Limperopoulos C, Shevell M, et al. Health and well-being of children with congenital cardiac malformations, and their families, following open-heart 
surgery. Cardiol Young 2006;16:157-64.

23. Lee S, Yoo JS, Yoo IY. Parenting stress in mothers of children with congenital heart disease. Asian Nurs Res (Korean Soc Nurs Sci) 2007;1:116-24.

24. Brosig CL, Mussatto KA, Kuhn EM, et al. Psychosocial outcomes for preschool children and families after surgery for complex congenital heart disease. Pediatr Cardiol 2007;28:255-62.

25. Gardner FV, Freeman NH, Black AM, et al. Disturbed mother-infant interaction in association with congenital heart disease. Heart 1996;76:56-9.

26. Darke PR, Goldberg S. Father-infant interaction and parent stress with healthy and medically compromised infants. Infant Behav Dev 1994;17:3-14.

27. Goldberg S, Simmons RJ, Newman J, et al. Congenital heart disease, parental stress, and infant-mother relationships. J Pediatr 1991;119:661-6.

28. Lan SF, Mu PF, Hsieh KS. Maternal experiences making a decision about heart surgery for their young children with congenital heart disease. J Clin Nurs 2007;16:2323-30.

29. Maxwell GM, Gane S. The impact of congenital heart disease upon the family. Am Heart J 1962;64:449-54.

30. Pinelli JM. A comparison of mothers' concerns regarding the care-taking tasks of newborns with congenital heart disease before and after assuming their care. J Adv Nurs 1981;6:261-70.

31. Docherty SL, Miles MS, Holditch-Davis D. Worry about child health in mothers of hospitalized medically fragile infants. Adv Neonatal Care 2002;2:84-92.

32. Brosig CL, Whitstone BN, Frommelt MA, et al. Psychological distress in parents of children with severe congenital heart disease: the impact of prenatal versus

Cite this article as: Biber S, Andonian C, Beckmann J, Ewert P, Freilinger S, Nagdyman N, Kaemmerer H, Oberhoffer R, Pieper L, Neidenbach RC. Current research status on the psychological situation of parents of children with congenital heart disease. Cardiovasc Diagn Ther 2019;9(Suppl 2):S369S376. doi: 10.21037/cdt.2019.07.07 postnatal diagnosis. J Perinatol 2007;27:687-92.

33. Abidin RR. Parenting Stress Index (PSI). Charlottesville: Pediatric Psychology Press, 1990.

34. Goldberg S, Morris P, Simmons RJ, et al. Chronic illness in infancy and parenting stress: A comparison of three groups of parents. J Pediatr Psychol 1990;15:347-58.

35. Cohn JK. An Empirical Study of Parents' Reaction to the Diagnosis of Congential Heart Disease in Infants. Soc Work Health Care 1996;23:67-79.

36. Azhar AS, AlShammasi ZH, Higgi RE. The impact of congenital heart diseases on the quality of life of patients and their families in Saudi Arabia: biological, psychological, and social dimensions. Saudi Med J 2016;37:392-402.

37. Lawoko S, Soares JJ. Social support among parents of children with congenital heart disease, parents of children with other diseases and parents of healthy children. Scand J Occup Ther 2003;10:177-87.

38. Hoekstra-Weebers JE, Jaspers JP, Kamps WA, et al. Psychological adaptation and social support of parents of pediatric cancer patients: A prospective longitudinal study. J Pediatr Psychol 2001;26:225-35.

39. Almesned S, Al-Akhfash A, Mesned AA. Social impact on families of children with complex congenital heart disease. Ann Saudi Med 2013;33:140-3.

40. Wiener L, Battles H, Zadeh S, et al. The perceived influence of childhood cancer on the parents' relationship. Psychooncology 2017;26:2109-17.

41. Burns W, Péloquin K, Sultan S, et al. A 2-year dyadic longitudinal study of mothers' and fathers' marital adjustment when caring for a child with cancer. Psychooncology 2017;26:1660-6. 